\title{
The EDI 320
}

As clinicians we often attempt to document spinal mobility, either as part of our return-to-work evaluations or for disability determination. However, spinal mobility is particularly difficult to evaluate because it is a compound movement between multiple joints in the spine and the hips. The use of inclinometry to quantify spinal mobility evolved in the mid-1980s out of a need to increase the accuracy and objectivity of these measurements. The EDI is a computerized inclinometer that is designed to measure, display, and save joint motion. This instrument can be used to measure extremity motion as well as spinal mobility. In the clinical setting, however, it is more likely to be used for measuring spinal mobility.

The EDI 320 has three modes of operation: single reference, continuous, and compound. The single reference mode is used primarily for measuring extremity joint range when the limb segment is moving from one reference position to another. This mode can also be used to measure relative angles between body segments on $\mathrm{x}$-ray film. In this single reference mode, the EDI 320 saves the measurement for 5 minutes after it is taken. The continuous mode is similar to the single reference mode except that the measurement is not recorded and saved at the end of the measurement but is read and recorded by the examiner. The compound mode is used primarily for measuring motion that occurs over several joints, such as flexion/extension, side bending, and rotation of the spine. In measuring lumbar motion, for example, the motion occurring at the hip is subtracted from the total motion to obtain the motion occurring in the lumbar spine. This method of measuring spinal motion was described and popularized by Mayer et al., (1984), Keeley et al., (1986), Mellin (1986), and others. The EDI 320 automates, digitally displays, and saves these measurements.

The EDI 320 sells for just under $\$ 500.00$ and consists of four components: a display unit, a hand-held unit, a long-bone attachment, and a lateral interface. The display unit contains the digital display of the range of motion measures, control switches, and the battery compartment. The display unit can either rest on a table or be mounted on a wall. The hand-held unit is the component that is placed on the patient's body and used to take the range of motion readings. The hand-held unit is attached to the display unit by a 10-foot coiled cord. The long-bone attachment is a straight edge that attaches to the hand-held unit to enhance stability of the unit during extremity measurements. The lateral interface attaches to the hand-held unit for measuring side bending of the trunk. The EDI is powered by a nickel cadmium rechargeable battery that has a life of approximately 12 hours and requires approximately 12 hours to recharge. A low-battery message will appear on the display unit if the battery is low.

A procedure manual accompanies the EDI 320. In general, the procedure manual is fairly well written and is accompanied by good illustrations and diagrams. Some fine tuning of the written instructions, however, might improve clarity. The testing patterns for each of the trunk motions lack a description of position of the arms during the testing procedure. Arm position can affect both the execution and the end range of spinal mobility, especially for extension and side bending of the lumbar spine. Therefore, a description of arm and hand placement during trunk movements should be included in the instructions. Verbal instructions to the patient for these more complex measurements should also be included.

To date, there is only one unpublished study that addresses reliability of the EDI 320 in measuring flexion and extension (Allen, Douglas, Main, and Gray, 1988). Because this study has not been published in a refereed journal, judging the adequacy of the methodology is difficult. Reliability of measuring side bending and rotation using the EDI 320 has not been addressed. Validity studies

WORK 1993; 3(3):79-80

Copyright (C) 1993 by Andover Medical 
comparing EDI measurements to flexion and extension radiographs are also lacking. Other studies addressing reliability and validity of inclinometry have been performed with noncomputerized inclinometers (Keeley et al., 1988; Mellin, 1986; Mayer et al., 1984). These inclinometers are circular disks with a weighted pendulum and are read by noting the location of the pendulum on the dial. They have the advantage of being very inexpensive and the disadvantage of being more cumbersome to use than the EDI. Reliability of these noncomputerized inclinometers has been found to be good for measures of flexion, extension, and rotation (Mellin, 1986; Mayer et al., 1984). For measuring side bending, however, their reliability was found to be fair (Mellin, 1986). Validity has been evaluated for flexion and extension only (Mayer et al., 1984). Radiographic flexion/extension films were compared to inclinometer measurements on 12 subjects. No significant differences were found between radiographs and inclinometer measurements. Obviously, further research, published in a refereed journal that specifically addresses the reliability and validity of the EDI 320 is needed.

In summary, the EDI 320 is a computerized inclinometer used for measuring joint range of motion. The digital display, internal calculation of neutral, and its ability to save measurements make it easier to use than noncomputerized inclinometers. Until comprehensive reliability and validity studies are performed and published in a refereed journal, however, the added expense of the EDI may be difficult to justify.

Deborah Lechner, $M S, P T$

\section{REFERENCES}

Allen, M. E., Douglas, L., Main, C., Gray, K. (1988). Examining lumbar and sacral movement patters using hand held electrogoniometer (EDI-320). Unpublished data, SFU Kinesiology. April.

Keeley, J., Mayer, T. G., Cox, R., et al. (1986). Quantification of lumbar function. Part 5: Reliabilityof-motion measures in the sagittal plane and an In Vivo torso rotation measurement technique. Spine 11, 31-35.

Mellin, G. (1986). Measurement of thorocolumbar posture and mobility with a myrin inclinometer. Spine 11, 759-762.

Mayer, T. G., Tencer, A. F., Kristoferson, S., et al. (1984). Use of noninvasive techniques for quantification of spinal range-of-motion in normal subjects and chronic low-back dysfunction patients. Spine 9 , 588-595. 